\title{
Fractal geometry of spin-glass models
}

\author{
J. F. Fontanari \\ Instituto de Fúsica de São Carlos, Universidade de São Paulo, \\ Caixa Postal 369, 13560-970 São Carlos SP, Brazil \\ P. F. Stadler \\ Institut für Theoretische Chemie, Universität Wien, Währingerstraße 17, A-1090 Wien, Austria \\ The Santa Fe Institute, 1399 Hyde Park Road, Santa Fe, NM 87501, USA
}

\begin{abstract}
Stability and diversity are two key properties that living entities share with spin glasses, where they are manifested through the breaking of the phase space into many valleys or local minima connected by saddle points. The topology of the phase space can be conveniently condensed into a tree structure, akin to the biological phylogenetic trees, whose tips are the local minima and internal nodes are the lowest-energy saddles connecting those minima. For the infinite-range Ising spin glass with $p$-spin interactions, we show that the average size-frequency distribution of saddles obeys a power law $\langle\psi(w)\rangle \sim w^{-D}$, where $w=w(s)$ is the number of minima that can be connected through saddle $s$, and $D$ is the fractal dimension of the phase space.

PACS 75.10.Nr (principal), 87.23. Kg
\end{abstract}

The resemblance between models of adaptive evolution [1] and disordered spin systems [2] is certainly not coincidental. In fact, two key features that any successful model of biological evolution ought to possess are stability and diversity, i.e., exactly the properties responsible for the complex thermodynamics of spin glasses [3]. It is not a surprise therefore, that many of the tools and concepts of the statistical mechanics of disordered systems have been applied to the study of the evolutionary process. In this contribution we show that such interchange can be profitable to statistical mechanics too, in that a great deal of information about the phase space of spin-glass models can be condensed into a tree structure, in a standard procedure widely used in taxonomy and molecular phylogenetics [4]. As in the biological case, the geometric properties of this tree can be used to characterize the disordered system quantitatively.

The main unifying concept in the investigations of the physics of disordered systems and evolutionary change is probably the notion of fitness or energy landscape. The concept of neighborhood among genotypes (configurations), typically defined such that point mutations interconvert neighbors, allows us to view the set of all genotypes as the vertices of a graph with edges connecting neighboring configurations. A fitness landscape is then obtained by assigning a fitness value to each vertex. An explicit connection between those two research fields is obtained in the case of populations of asexually reproducing haploid organisms evolving on rugged fitness landscapes. In this case the genotypes are often modeled by configurations of $N$ Ising spins $s=\left(s_{1}, \ldots, s_{N}\right)$ with $s_{i}= \pm 1$ so that a point mutation corresponds to a single spin flip. In the simplest case, evolutionary adaptation is described as an "adaptive walk" on the fitness landscape [1], whose statistical mechanics equivalent is the zero-temperature Glauber dynamics. The fitness function assigns a random numerical value to each one of the $2^{N}$ spin configurations. In this work we consider the $p$ - spin landscapes [5:

$$
\mathcal{H}_{p}(s)=-\sum_{1 \leq i_{1} \leq i_{2} \ldots \leq i_{N} \leq N} J_{i_{1} i_{2} \ldots i_{N}} s_{i_{1}} s_{i_{2}} \ldots s_{i_{N}}
$$

where the $J_{i_{1} i_{2} \ldots i_{N}}$ are statistically independent Gaussian distributed random variables with mean zero and variance $p ! /\left(2 N^{p-1}\right)$. The $p$-spin models form a class of tunably rugged landscapes similar to Kauffman's Nkmodel [1], which is not only more appealing to statistical mechanics but also is a more natural basis of landscape theory [6]. In fact, for $p=2$ the Hamiltonian $\mathcal{H}_{p}$ reduces to the SK model [7] which exhibits a large number of highly correlated local minima, while the limit $p \rightarrow \infty$ corresponds to the random energy model (REM) [5] and yields an extremely rugged, uncorrelated landscape. Like the Nk-model, $p$-spin landscapes have been used repeatedly to model evolutionary processes, see e.g. [3, 8].

The scenario that emerges from the replica approach to disordered spin systems is that the phase space composed of the $2^{N}$ spin configurations is broken into many valleys [2]. The ease with which one valley can be reached from another one depends on the saddle points connecting them. More specifically, the energy of the lowest saddle point separating two local minima $x$ and $y$ is

$$
E[x, y]=\min _{\mathbf{p} \in \mathbb{P}_{x y}} \max _{z \in \mathbf{p}} \mathcal{H}_{p}(z)
$$

where $\mathbb{P}_{x y}$ is the set of all paths $\mathbf{p}$ connecting $x$ and $y$ by a series of subsequent spin-flips (or point mutations). The saddle-point energy $E[.,$.$] is an ultrametric dis-$ tance measure on the set of local minima, see e.g. [9].

Let us assume for a moment that the energy function is non-degenerate, i.e., $\mathcal{H}_{p}(x) \neq \mathcal{H}_{p}(y)$ whenever $x \neq y$. This is true for generic $p$-spin models with odd interaction order $p$. Then there is a unique saddle point $s=s(x, y)$ connecting $x$ and $y$ characterized by $\mathcal{H}_{p}(s)=E[x, y]$. 


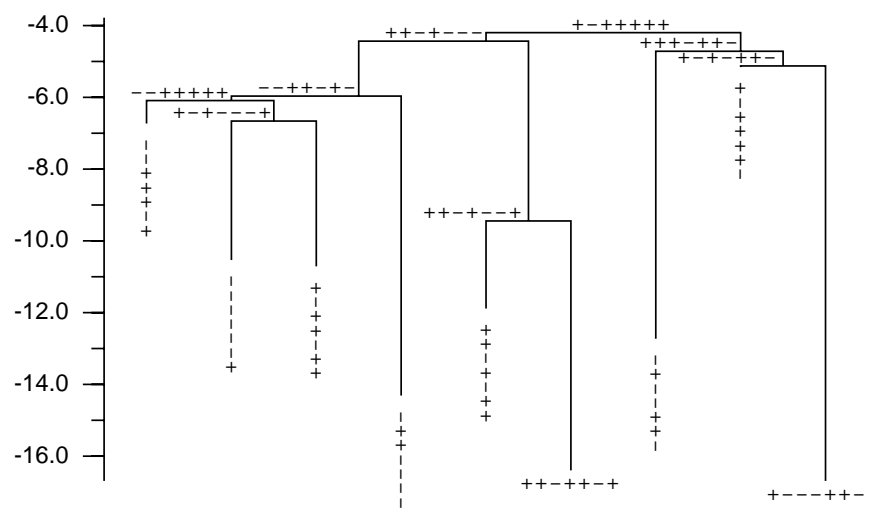

FIG. 1: Barrier tree for a 3-spin model with $N=7$. The 9 minima (tips) and 8 connecting saddle points (internal nodes) are labeled by their spin configurations.

Note that this definition of saddle point is more restrictive than in differential geometry where saddles are not required to separate local optima [10]. To each saddle point $s$ there is a unique collection of configurations $B(s)$ that can be reached from $s$ by a path along which the energy never exceeds $\mathcal{H}_{p}(s)$. In other words, the configurations in $B(s)$ are mutually connected by paths that never go higher than $\mathcal{H}_{p}(s)$. This property warrants to call $B(s)$ the valley or basin below the saddle $s$. Furthermore, suppose that $\mathcal{H}_{p}(s)<\mathcal{H}_{p}\left(s^{\prime}\right)$. Then there are two possibilities: if $s \in B\left(s^{\prime}\right)$ then $B(s) \subseteq B\left(s^{\prime}\right)$, i.e., the basin of $s$ is a "sub-basin" of $B\left(s^{\prime}\right)$, or $s \notin B\left(s^{\prime}\right)$ in which case $B(s) \cap B\left(s^{\prime}\right)=\emptyset$, i.e., the valleys are disjoint. This property arranges the local minima and the saddle points in a unique hierarchical structure which is conveniently represented as a tree, termed barrier tree (see Fig. 1).

In principle, barrier trees can be computed by means of the following simple recursive procedure: The tips of the tree are the local minima. The parent of tip $x$ is the lowest-energy saddle point $s$ that connects $x$ to another local minimum. Analogously, the parent of a saddle point $s$ is another saddle point $s^{\prime}$ that connects $s$ to a local minimum $z$ that is not contained in the basin below $s$, i.e., $z \notin B(s)$. The "root" of the resulting tree is the saddle point $s^{*}$ with the highest energy, since by definition all local minima are contained in $B\left(s^{*}\right)$. Note that the subtree $\mathfrak{T}(s)$ that has the saddle $s$ as its root has exactly the local minima in $B(s)$ as its tips. The exact calculation of the barrier tree is a highly challenging computational problem and only recently some progress in that direction has been achieved, mainly in the context of RNA and protein folding [11, 12] (see also [13]). The reason is that, unless one has sufficient a priori knowledge on the landscape, it is necessary to generate the complete landscape in order to find all local minima. Even for very small system sizes a simple-minded exhaustive search approach to evaluating Eq. (2) would be hopeless as one must calculate all paths connecting all pairs of minima. In this contribution we use the program package barriers-0.9

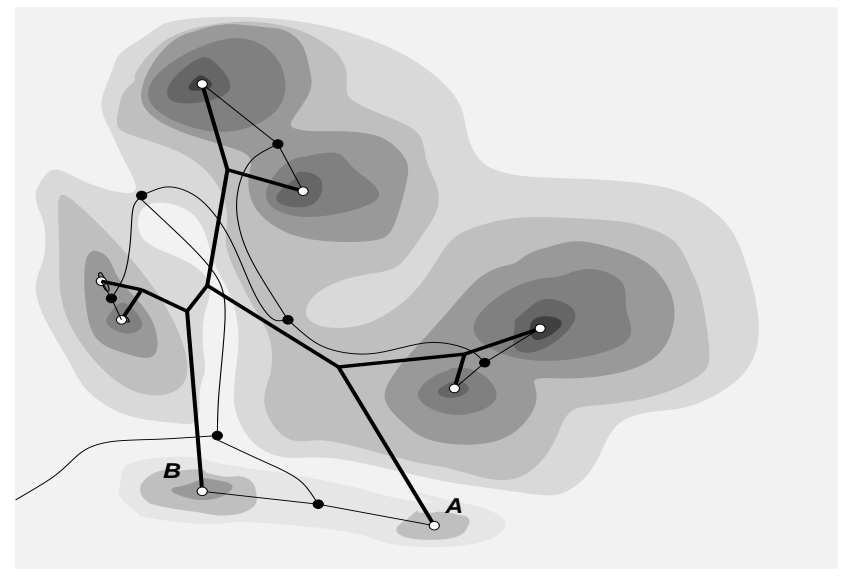

FIG. 2: Unrooted phylogenetic tree of extant species (white dots) obtained from the minimum fitness paths, shown by thin lines with saddle points (ancestors) indicated by black dots, does not necessarily coincide with the tree obtained from clustering methods that are based on sequence similarity, shown here with thick lines. The gray intensity is proportional to the fitness value.

to construct the barrier tree from an energy sorted list of spin configurations in linear time. The algorithm explicitly constructs the basins $B(s)$ and subtrees $\mathfrak{T}(s)$ [11.

The barrier tree can be viewed as a phylogenetic tree with a single common ancestor at the root. The evolutionary process leading to the extant species (i.e., the tips of the tree) is an adaptive walk on the rugged fitness landscape (11). Interestingly, according to definition (2) a subtree connecting two tips corresponds to the evolutionary path of minimum fitness cost which could be regarded as a generalization of the maximum parsimony principle [4 to rugged fitness landscapes. This differs from the usual approach in molecular biology, where a flat fitness landscape and a diffusive behavior in sequence space is assumed to justify the reconstruction of phylogenetic trees based solely on sequence similarity, i.e., configurational overlap. In Fig. 2 we give an example in which the distance-based tree and the barrier tree have different topologies. In particular, the species labeled $A$ and $B$ are much closer in the barrier tree than in the maximum parsimony tree. It is interesting to note that barrier trees can be defined in a meaningful way also for continuous energy surfaces. Their nodes are the local minima and the saddle points satisfying Eq. (2), while the edges can be associated with saddle connections along which the energy varies monotonically.

The definition of barrier trees becomes more complicated if the energy function is degenerate as in the case of $p$-spin models with even $p$, where $\mathcal{H}_{p}(s)=\mathcal{H}_{p}(-s)$. The appropriate definition of the barrier tree is obtained by identifying the saddle points $s$ and $s^{\prime}$ with the same interior node of the tree provided (i) they have the same energy and (ii) they are connected by a path along which the energy does not exceed $\mathcal{H}_{p}(s)=\mathcal{H}_{p}\left(s^{\prime}\right)$. In the nondegenerate case the trees are almost always binary. De- 
generacies can occur also for geometric reasons since the same saddle point can connect more than two basins. One may, however, "expand" a non-binary interior node into a sequence of binary nodes at the same height. We use this technical trick here to simplify the computations. This procedure is justified because it affects only the saddle points above the saddle $\tilde{s}$ that connects the groundstate and its mirror image, i.e., it affects only the nodes with large basins, far beyond the regime where the tree exhibits self-similarity.

One important aggregate characteristic of a tree is the size-frequency distribution of its saddles or subtrees $\psi(w)$, where the size $w=w(s)$ is, in the simplest case, just the number of local minima or tips in $B(s)$. It is instructive to consider first a few examples of simple ideal trees for which $\psi(w)$ can be calculated analytically. E.g., a symmetric binary tree of depth $m \geq 1$ has $2^{m}-1$ nodes, of which $2^{m-1}$ are tips and the remaining $2^{m-1}-1$ are saddles. It can be easily shown that there are $2^{m-k-1}$ saddles with sizes $w=2^{k} ; k=1, \ldots, m-1$, so that

$$
\psi(w)=\left\{\begin{array}{cc}
\frac{1}{w}\left(1-2^{1-m}\right) & \text { if } \log _{2} w \in \mathbb{N} \\
0 & \text { if } \log _{2} w \notin \mathbb{N} .
\end{array}\right.
$$

The other extreme is the asymmetric binary tree in which every left child is a tip. There are $2 m-1$ nodes: $m$ tips and one saddle with size $w, 1 \leq w \leq m-1$. Hence

$$
\psi(w)=\frac{1}{m-1} \quad \text { for } 2 \leq w \leq m
$$

We turn now to the analysis of the complex trees associated to the random energy function (1) as produced by the program barriers-0.9. In this case the average number of tips increases exponentially with the number of spins $\mathrm{e}^{\alpha_{p} N}$, where $\alpha_{p}$ increases from $\alpha_{2}=0.199$ to $\alpha_{\infty}=\ln 2$ 2]. Log-log plots of the average size-frequency distributions of subtrees for particular $p$-spin models and the REM are shown in Fig. 3. In all cases the data are very well fitted by straight lines with negative slopes in the regime of high frequencies, suggesting then a power law form

$$
\langle\psi(w)\rangle \sim w^{-D}
$$

where $D$ can be viewed as the fractal dimension of the barrier tree and hence of the phase space of the disordered system. This result points out a very large number of subtrees with a few tips and a very small number of subtrees with many tips. Moreover, it implies that there is no characteristic number of tips within subtrees. As illustrated by the gray data points in the panel for the REM, the same scaling law seems to hold true for the size-frequency distribution of a single instance of the landscape as well. The asymmetric scattering of points observed in the low frequency regime, i.e., $\langle\psi(w)\rangle \ll 1$, is due to the few high energy sadddle points near the
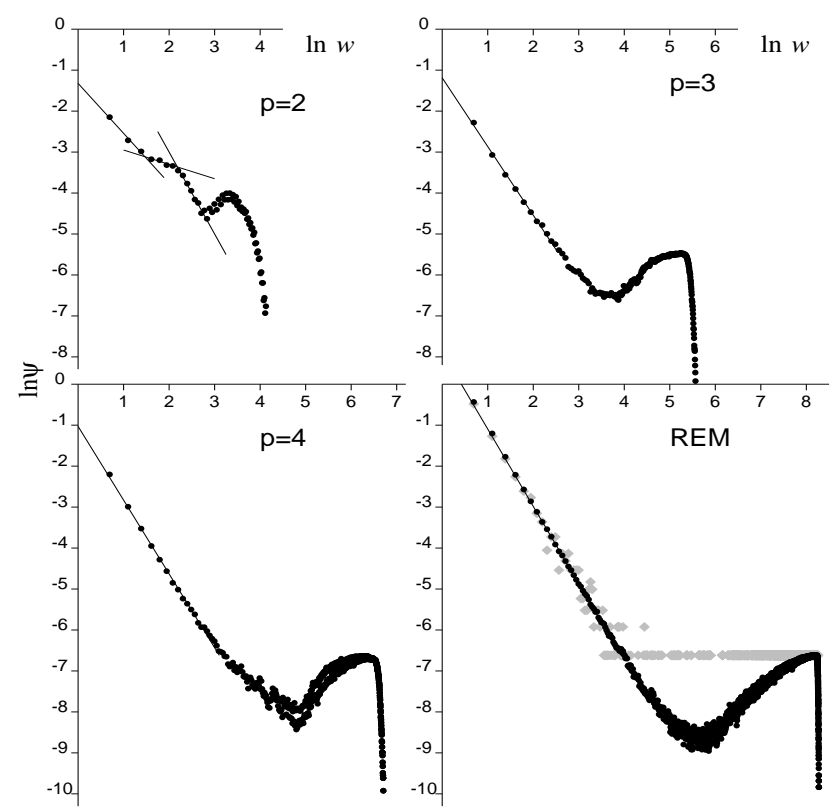

FIG. 3: Log-log plots of the average frequency of subtrees (saddle points) with different numbers of tips (minima) for the $p$-spin landscapes with $N=18$, and the REM with $N=16$, averaged over 300 landscapes each. For the REM, a sizefrequency distribution for a single landscape is shown as gray squares to demonstrate the scatter.

root of the tree. It is interesting to note that many frequency distributions of taxonomic units containing various numbers of subunits (e.g., species per genus) were found to be well described by power laws 14. It should be stressed that the scaling law (5) is by no means a mere consequence of the existence of an underlying tree structure, as it is clear from the asymmetric binary tree example discussed before as well as from the study of discrete branching processes which generate different forms of size-frequency distributions 15$]$.

The average size-frequency distribution for the SK model displays a rather distinct behavior pattern which seems to indicate the existence of two different types of self-similar structures at different levels of the tree. These structures are characterized by straight lines with distinct slopes, being joined by a short, almost flat curve corresponding to a crossover regime between the dominant structures. The first slope appears to be around $D_{1} \approx 1.4$, the second slope is $D_{2} \approx 2$. These results, however, must be taken with caution since the size-frequency statistics is greatly impaired by the fact that the trees are typically very small in this model. For instance, for $N=18$ the average number of tips is about 50 in the SK model as compared to the $\sim 10^{5}$ tips in the REM.

In addition to being a global measure for the characterization of rugged landscapes, to be contrasted with local measures such as the correlation length [6, 16], the parameter $D$ also yields a measure of diversity since $D$ is higher in systems where subtrees with one or a few tips 


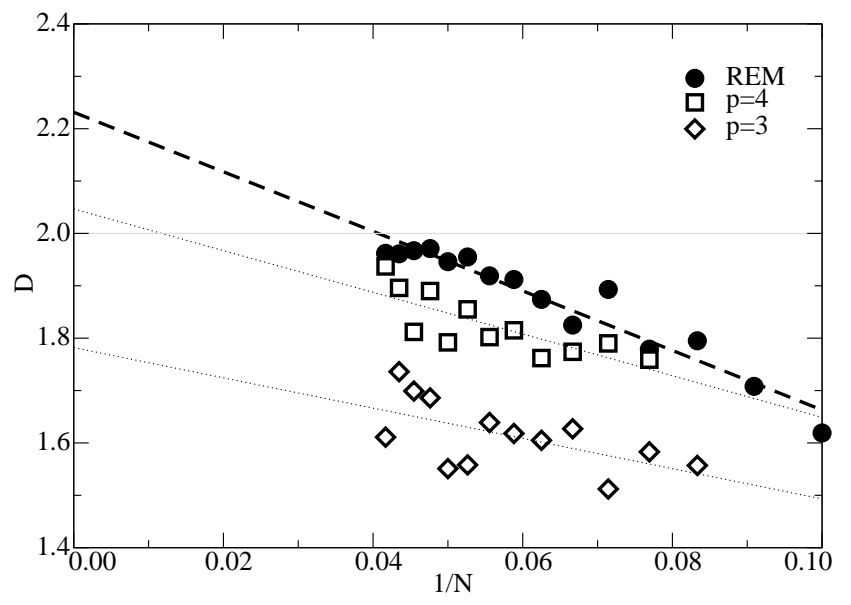

FIG. 4: Dependence of the exponents $D$ on the reciprocal of the number of spins $N$. The data for $p=2$ do not allow for the unambiguous estimate of an exponent $D$.

are more numerous. An attempt to estimate the fractal dimension $D$ for infinite system sizes is presented in Fig. 4. From these data one cannot discard the possibility that for very large systems the exponents will converge to $D=2$ independently of $p$, in which case the value of $D$ might be seen as an index that characterizes the universality class of the $p$-spin landscapes. Since the exact construction of barrier trees is at present feasible for systems of sizes up to $N=24$ only, the estimate of $D$ for larger systems must resort to a stochastic approach, probably in the spirit of the coalescent theory of population genetics [4], which focuses on the genealogy of a few sampled individuals rather than on the family tree of the entire population.

The replica theory predicts a similar hierarchical structure for the space of pure states, consisting of clusters within clusters. In particular, the probability density that a cluster has weight $W$ (roughly the fraction of pure states within it) is [2]

$$
f(W)=\frac{W^{y-2}(1-W)^{-y}}{\Gamma(y) \Gamma(1-y)}
$$

which for small weights reduces to a power law $f(W) \sim$ $W^{y-2}$. Here $y \in(0,1)$ is a complicated function of the physical parameters $p$ and the temperature $T$. For instance, for the REM one has $y=1-T / T_{c}$ where $T_{c}=(4 \ln 2)^{-1 / 2}$. As the replica pure states space is a rather abstract construct (e.g., only a few low-energy local minima are pure states and the definition of clus- ters does not involve the notion of saddle points) a direct comparison with our results is not evident; albeit it is highly desirable since we are not aware of any attempt to verify Eq. (6) via numerical simulations.

Acknowledgements. The work of J.F.F. is supported in part by CNPq and FAPESP, Proj. No. 99/09644-9. This research was performed during a stay at ZIF in Bielefeld in May 2001 as part of the working group The Sciences of Complexity: From Mathematics to Technology to a Sustainable World.

[1] S. A. Kauffman, The Origins of Order (Oxford University Press, Oxford, 1993).

[2] M. Mézard, G. Parisi and M. A. Virasoro, Spin Glass Theory and Beyond (World Scientific, Singapore, 1987).

[3] P. W. Anderson, Proc. Natl. Acad. Sci. USA 80, 3386 (1983).

[4] M. Nei, S. Kumar, Molecular Evolution and Phylogenetics (Oxford University Press, Oxford 1999). R. D. M. Page, E. C. Holmes, Molecular Evolution: A Phylogenetic Approach (Blackwell Science Inc, 1998).

[5] B. Derrida, Phys. Rev. B 24, 2613 (1981).

[6] P. F. Stadler, J. Math. Chem. 20, 1 (1996).

[7] D. Sherrington and S. Kirkpatrick, Phys. Rev. Lett. 35, 1792 (1975).

[8] C. Amitrano, L. Peliti and M. Saber, J. Mol. Evol. 29, 513 (1989). D. S. Rokhsar, P. W. Anderson and D. L. Stein, J. Mol. Evol. 23, 119 (1986).

[9] R. Rammal, G. Toulouse and M. A. Virasoro, Rev. Mod. Phys. 58, 765 (1986).

[10] A. M. Vertechi, M. A. Virasoro, J. Phys. France, 50, 2325 (1989).

[11] C. Flamm, W. Fontana, I. Hofacker and P. Schuster, RNA 6, 325 (2000).

[12] P. Garstecki, T. X. Hoang and M. Cieplak, Phys. Rev. E 60, 3219 (1999).

[13] O. M. Becker and M. Karplus, J. Chem. Phys. 106, 1495 (1997). F. F. Ferreira, J. F. Fontanari and P. F. Stadler, J. Phys. A 33, 8635 (2000). T. Klotz and S. Kobe, J. Phys. A 27, L95 (1994).

[14] J. C. Willis, Age and Area (Cambridge University Press, Cambridge 1922). B. Burlando, J. Theor. Biol. 146, 99 (1990); 163, 161 (1993).

[15] J. Chu, C. Adami, Proc. Natl. Acad. Sci. USA 96, 15017 (1999).

[16] E. D. Weinberger Biol. Cybern. 63, 325 (1990). W. Fontana, T. Griesmacher, W. Schnabl, P. F. Stadler, P. Schuster, Monatsh. Chem. 122, 795 (1991). E. D. Weinberger and P. F. Stadler, J. Theor. Biol. 163, 255 (1993). 